\title{
Cross-generational effects of male reproductive success and offspring immunocompetence in Drosophila melanogaster
}

\author{
Ashley Guncay $^{\text {ab }}$, Thiropa Balasubramaniam ${ }^{a}$, Katie Plagens ${ }^{a}$, Joel Weadge ${ }^{a}$, and Tristan A.F. Long ${ }^{a \star}$ \\ ${ }^{a}$ Department of Biology, Wilfrid Laurier University, 75 University Avenue West, Waterloo, ON N2L 3C5, \\ Canada; ${ }^{\mathrm{b}}$ Department of Medical Genetics, University of Alberta, 8613114 Street, Edmonton, \\ AB T6G 2H7, Canada \\ *tlong@wlu.ca
}

\begin{abstract}
In some species where males make no direct contribution to a female's lifetime reproductive success, females choose mates based on the indirect benefits manifested in their offspring. One trait that may be subject to this sexual selection is immunocompetence (the ability to mount an immune response following exposure to pathogens); however, the results of previous work on its link to male attractiveness have been ambiguous. Herein we examine the life history consequences of mating with males with a history of failure or success in reproductive competitions in Drosophila melanogaster. By examining egg-to-adult survival, body weights, and bacterial loads of offspring reared in either the absence or presence of a bacterial pathogen, we were able to examine whether sire reproductive success was associated with their offsprings' ability to respond to an immunological challenge and other life history traits. Our results are partially consistent with the predictions of the "immunocompetence handicap hypothesis": competitively successful males ("studs") sire male offspring that are better able to handle an immunological challenge than those sired by competitively unsuccessful males ("duds"). However, our assay also revealed the opposite pattern in female offspring, suggestive of the complicating presence of alleles with sexually antagonistic effects on the expression of this important life history trait.
\end{abstract}

Key words: mate choice, sexual selection, sexual conflict, indirect benefits, multi-generational, immunocompetence, immune challenge, Drosophila melanogaster

\section{Introduction}

In many species where males provide no parental care or other direct resources to their mates, females often exhibit preferences for mates that are "attractive" and (or) of superior intra-sexual competitive ability (see Andersson 1994, pp. 124-142). Whether these preferences are maintained because females obtain indirect genetic benefits from being choosy is one of the most debated questions amongst evolutionary biologists (Kirkpatrick and Ryan 1991; Kokko et al. 2006; Qvarnström et al. 2006). Hamilton and Zuk (1982) hypothesized that female preferences evolve because elaborate traits in males reveal important information about the underlying genetic "quality" of their immune system; by being discriminating, females are able to produce offspring with greater potential for 
immunocompetence. This "good genes" model of sexual selection (Andersson 1994) has been subsequently expanded and modified (Folstad and Karter 1992; Sheldon and Verhulst 1996; Rolff 2002) to include a mechanistic explanation for why sexual signals act as "honest indicators" (sensu Zahavi 1975): that there are unavoidable physiological conflicts that arise in the construction and maintenance of both ornaments and immune response mechanisms (due to the antagonistic action of hormones and (or) limits on energetic and nutritional resources).

If resources are the limiting factor, then both immune defense and sexual signals should be thought of as condition-dependent life history traits, the absolute and relative expression of which will depend on both the individual's accumulation and allocation of resources (Rowe and Houle 1996). If there is a high level of genetic variation for resource acquisition in a population, then individuals of high genetic "quality" (and their offspring) will be able to devote more resources to building and maintaining both immune systems and sexual advertisements than individuals of low "quality". In contrast, if there is relatively more genetic variation for resource allocation, then trade-offs and negative correlations between these life history traits will be revealed (van Noordwijk and de Jong 1986; Houle 1991; Westneat and Birkhead 1998). Genetic variation for allocation may be especially important if the sexes have different optimal levels of investment into immune function and other life history traits (due to sex-specific fitness-maximizing strategies). Males may benefit from greater investment into traits that increase attractiveness (and thus mating success), and be selected to invest less into immunocompetence than females (Zuk 1990; Rolff 2002). In cases of sexual conflict, alleles that may be beneficial when expressed in one sex may be maladaptive when expressed in members of the opposite sex (Holland and Rice 1998; Chippindale et al. 2001; Pischedda and Chippindale 2006).

One way in which the relationships between life history traits can be explored is by comparing the phenotypes of offspring after one or multiple generations of divergent selection on one life history trait (Zera and Harshman 2009). An early example of this approach was that of Kurtz and Sauer (1999), who compared the immunocompetence of offspring sired by male scorpionflies (Panorpa vulgaris) that differed in the degree of ornament elaboration and found a small immune benefit associated with females being choosy. More recently, a trio of experimental evolution studies, using the model species Drosophila melanogaster, have examined life history trade-offs from different perspectives. In this species, males compete intra-sexually, as well as engage in elaborate inter-sexual courtships of females, using a wide range of chemical, visual, and acoustic signals (Hall 1994; Greenspan and Ferveur 2000). As in many insect species, D. melanogaster does not possess an adaptive immune system and must rely on an innate system which confronts bacterial and fungal infections through the production of antimicrobial peptides, and a cellular system that primarily uses phagocytosis and encapsulation to combat parasitoids (Broderick and Lemaitre 2009). The construction and maintenance of this immune system can be costly (Kraaijeveld and Wertheim 2009), and studies of $D$. melanogaster are proving to be central to understanding immune function in a wide variety of animal taxa (Khush and Lemaitre 2000; Hoffmann 2003). In the first study, Rolff and Kraaijeveld (2003) selected for increased pathogen resistance in replicate experimental populations by exposing flies to the parasitic wasp Asobara tabida. This selection resulted in the evolution of greater pathogen resistance, but at the cost of decreased larval competitiveness when food resources were limited (Kraaijeveld and Godfray 1997; Kraaijeveld et al. 2001). Despite no significant difference in body size between flies from unselected control populations and those from experimental populations, flies in the latter group had higher mating success (by achieving more matings in direct competition with males from a tester stock and by mating more rapidly) than did flies from the former group (Rolff and Kraaijeveld 2003). The second relevant study by McKean and Nunney (2008) took a different selective approach by creating experimental populations that experienced greater levels of sexual selection (by artificially creating male-biased sex ratios in every generation of culture). They found that relative to flies from the control populations, experimental flies were larger, 
developed more slowly, and males were more successful at obtaining matings. However, both male and female flies in the experimental populations were less resistant to immunological challenge from Escherichia coli than those from the control populations. More recently, Modak et al. (2009) compared the resistance to $E$. coli in flies from populations that had been selected for faster development with those from corresponding control populations. They found that flies from the fast-selected lines exhibited higher rates of pathogen-induced mortality than did those from controls, suggestive of a genetic correlation between development rate and pathogen resistance. However, selection for faster development typically results in the evolution of smaller body size (e.g., Chippindale et al. 1997), which can also put male flies at a strong mating disadvantage (see Partridge et al. 1987). Overall, these various experiments suggest that the components of life history (mating success, body size, immunocompetence, and development rate) are linked. However, the magnitude and sign of these relationships remain unclear, as together these studies indicate that selection for increased male attractiveness may cause decreased immunocompetence (McKean and Nunney 2008), selection for increased immunocompetence may lead to increased male attractiveness (Rolff and Kraaijeveld 2003), and that decreased immunocompetence is correlated with a increased development rate and smaller body size (Modak et al. 2009).

Ultimately, support for a parasite-mediated sexual selection model to explain the evolution of some female preferences and elaborate male traits will emerge from a consensus of empirical tests of the genetic relationship between attractiveness, competitiveness, and immunocompetence. Here, we seek to add to this growing body of research by comparing the development, growth, and bacterial loads in D. melanogaster offspring that are sired by males differing in their mating success and comparing the performance of these offspring when experimentally exposed to the common insect pathogen Pseudomonas aeruginosa.

\section{Materials and methods}

\section{Experimental fly stocks and culturing protocol}

We measured male reproductive success and offspring performance using individual flies obtained from a large, outbred D. melanogaster population, Ives (hereafter "IV"), that originated from a collection made in South Amherst, Massachusetts, in 1975 and has been maintained on a standardized 2 week culture schedule since 1980 (Rose 1984). Briefly, this population is maintained at a large size ( 2800 adults/generation) in vials containing banana/agar/killed medium, in incubators set to $25^{\circ} \mathrm{C}$ on a $12 \mathrm{~h}$ light and $12 \mathrm{~h}$ dark diurnal light cycle and $60 \%$ humidity (Rose 1984; Long et al. 2006). We keep this population on a nonoverlapping 14 day culture cycle and propagate it by mixing the flies en masse between vials and placing them in roughly equal numbers into 28 "oviposition vials", each containing $10 \mathrm{ml}$ of standard media (and a light sprinkle of live yeast applied to the surface), for approximately $2 \mathrm{~h}$. We then manually cull the eggs laid during this period to a density of 100 eggs/vial, before returning them to the incubator to start the next generation of culture (Rose 1984; Long et al. 2006).

In addition to the flies obtained from the (wild-type) IV population, we also used flies from a second population, IV- $b w$, where all individuals exhibit a brown-eyed phenotype. This population was created by introgressing (through 10 rounds of backcrossing) the recessive brown-eyed genetic marker (" $b w$ ") into the IV genetic background. We culture IV-bw in exactly the same way as the IV population, and regular backcrossing ensures that it remains genetically similar to the IV population.

\section{Obtaining "stud" and "dud" males}

To assess cross-generational effects associated with sire reproductive success, we first needed to identify males of relatively high and low mating success, the offspring of which we could then examine 
(see Supplementary Material 1 for a graphical overview of the protocol). To accomplish this goal, we established three sets of 28 vials of IV eggs (temporally separated from each other by 2 days) and 28 vials of IV- $b w$ eggs (set up at the same time as the first set of IV vials). From the first set of vials we collected IV- $b w$ males, IV males and IV female adult progeny as virgins (within $8 \mathrm{~h}$ of their eclosion; Ashburner 1989), and held these flies in single sex groups (of $\sim 16$ adults/vial) for 2-3 days. We also collected virgin females from the second and third set of IV vials as they eclosed. In total, we obtained 250 virgin females from each set, which we then transferred into individual vials on the 12th day of their life cycle. At the same time, we created 250 vials, each containing a single IV male (target male) and an IV-bw male (competitor male), randomly combined for subsequent testing of the target male's relative mating success.

We used the virgin IV females from the first and second sets of IV vials to independently assay the relative mating success of the individual target males. This was done in sets of mating assays, which we conducted when flies were 14 days old (3-4 days post-eclosion), starting at 0900 (corresponding to "dawn" from the flies' perspective, when there is typically considerable reproductive activity). We combined males and females without anesthesia, mounted the vials horizontally in a quiet, well-lit room, and observed them for a period of $90 \mathrm{~min}$. We watched the trios of flies until a copulation event was observed. At that time, we noted the eye phenotype of the reproductively successful male (wild-type or brown-eyed). At the end of the trial, we removed and discarded the females from the vials and returned the males to the incubator to be used in a second trial 2 days later once their seminal fluids had a chance to replenish (Markow et al. 1978; Hihara 1981; Sirot et al. 2009). In the second trial, we combined the same pairs of males with IV virgin females collected from the second set of vials and we observed them for $90 \mathrm{~min}$, as in the manner described above. By this process, we were able to identify as per Rundle et al. (2007) sets of "stud" and "dud" males, where the former consisted of target wild-type males that had been twice successful at obtaining a copulation with a female, whereas the latter was composed of target wild-type males that had twice failed to mate. This protocol thus captures some aspects of both male attractiveness and inter-sexual competitive ability, with the caveat that random chance and male experience may have influenced specific outcomes. Before proceeding to the next stage of the experimental set up, we ensured that the "stud" and the "dud" groups were of the same size ( 40 individuals per group) to minimize the chance that any differences we would observe were due to differences in gene pool size.

\section{Experimental design/exposure to bacteria}

In the next step of the experiment, we removed the "stud" and "dud" males from their mating vials and distributed them across 10 vials (in groups of four males/vial) containing $\sim 15$ virgin females (obtained from the third set of IV vials). Vials contained ad libitum yeast and were left for a $48 \mathrm{~h}$ period to allow sufficient time for mating to occur and oviposition to begin. Next, we placed the flies into half-pint containers outfitted with $35 \mathrm{~mm}$ (diameter) Petri dishes containing grape juice-agar medium (Sullivan et al. 2000; with a scored surface to induce oviposition) for $18 \mathrm{~h}$ before being discarded. We then transferred the eggs laid on the media, in sets of 25 , into new vials containing $10 \mathrm{ml}$ of standard media, along with 75 IV- $b w$ eggs (of the same age) so that egg density in the vials resembled that of the base populations. In total, we established 80 vials containing "stud"-sired offspring and 80 vials containing "dud"-sired offspring for each replicate of this experiment. Immediately after the vials of eggs were set up, we experimentally exposed half of the vials containing "stud"-sired offspring and half of the vials containing "dud"-sired offspring to a bacterial pathogen, P. aeruginosa: a common, opportunistic, Gram-negative species of bacteria (Ryan and Ray 1994) that is a virulent pathogen of fruit flies (Boman et al. 1972; D'Argenio et al. 2001). Samples of Drosophila from both the lab and the field revealed the presence of Pseudomonas sp. amongst their bacterial communities (Chandler et al. 2011; Staubach et al. 2013), suggesting potentially widespread and ongoing ecological and co-evolutionary interactions between these groups. In our experiment, we used the 
facultative biofilm-producing PAO1 strain of P. aeruginosa (kindly supplied to JW by DE Ohman, Virginia Commonwealth University). Growth and handling of the pathogen followed the protocols described in Young et al. (2009) with minor modifications. Briefly, we cultured the P. aeruginosa in sterile Luria-Bertani (LB) medium overnight with shaking at $200 \mathrm{rpm}$ at $37^{\circ} \mathrm{C}$ and then diluted the culture to an $\mathrm{OD}_{600}$ of 1.0 with sterile $\mathrm{LB}$ media. We introduced the pathogen to the offspring using a biologically realistic method of exposure (Young et al. 2009) via the body of a dead infected virgin female. First we anesthetized the virgin females, and then infected them by pricking their abdomens with a size 00 insect pin that had been dipped into the live liquid culture. At the same time, we created a group of "control" virgin females by pricking them with an insect pin dipped into sterile LB medium. We pricked females the day prior to the experimental vial set-up (to permit time for the $P$. aeruginosa to replicate), and we decapitated the infected and control females immediately before they were introduced into the experimental and the control vials of eggs, respectively. In total, we established four experimental treatment combinations (pathogen-exposed "stud"-sired, pathogen exposed "dud"-sired, pathogen-control "stud"-sired, and pathogen-control "dud"-sired) each of which consisted of 40 replicate vials, which were placed in an incubator for a period of 14 days.

\section{Assessing survival, body size, and bacterial load}

At the end of 14 days, we removed all living flies from 30 of the 40 vials created for each treatment and tallied them by sex and eye colour to determine egg-to-adult survivorship. To ascertain the effect of paternity and (or) pathogen exposure on offspring development, we measured the weight of two randomly chosen wild-type males and females from each of the vials from which we measured survivorship (see Supplementary Material 1). These flies were immediately frozen and later dried in an oven overnight at $70^{\circ} \mathrm{C}$. Flies were then weighed to the nearest $0.001 \mathrm{mg}$ using a Sartorius SE2 Ultra Micro Balance (Sartorius AG, Gottingen, Germany).

To quantify representative bacterial loads for male and female offspring, under axenic conditions we collected sets of up to five males and five females from each of the remaining 10 replicate vials established for each treatment combination. We immediately placed same-sex groups of flies into microcentrifuge tubes containing $100 \mu \mathrm{l}$ of sterile LB media and two or three $1 / 8$ in. (diameter) Teflon balls (LabPure, Laboratory Products) that we had previously sterilized with $70 \%(v / v)$ ethanol. Next, we manually agitated the tubes (by repeatedly inverting them) for approximately $1 \mathrm{~min}$, followed by $1 \mathrm{~min}$ of vortexing, and then centrifuged them for $2 \mathrm{~min}$ (at $1790 \mathrm{~g}$ ) to spin all the solid contents to the bottom of the tube. We then used the supernatant in each vial to create a 10 -fold dilution series in LB media. We spread-plated a $20 \mu \mathrm{l}$ sample of dilutions $(1 / 10,1 / 100$, and $1 / 1000)$ on LB agar (1\%) and left the plate to incubate for $48 \mathrm{~h}$ at $25^{\circ} \mathrm{C}$ before counting bacterial colonies. We counted all plates containing between 30 and 300 colonies. From these counts, we estimated the individual bacterial load by calculating the colony forming units (CFU) per flies in each vial using standard protocols (Apidianakis and Rahme 2009).

\section{Secondary tests of relationship between immunocompetence and paternal mating success}

Following the completion of the experiment described above, we subsequently designed and undertook two additional post hoc experiments to test the plausibility of some of the functional explanations for the patterns of bacterial loads observed in the primary experiment. We designed these complementary assays to test whether (1) differences in offspring bacterial loads associated with sires of high and low mating success were also apparent in newly eclosed virgin flies, and (2) whether differences in bacterial load seen between daughters might be associated with differences in female mating rates. 
For the first of these tests, we repeated the pathogen-exposure protocol described above: categorizing males based on their success in two mating assays, generating "stud"- and "dud"-sired eggs, and then experimentally introducing $P$. aeruginosa into half of these vials. However, in this follow-up experiment we collected male and female offspring for use in our bacterial load assays as virgins (within $8 \mathrm{~h}$ of their eclosion from pupae, starting approximately day 9 of their culture cycle) rather than as sexually experienced, older adults. We immediately placed the flies into single-sex vials (containing fresh media) and sacrificed them for use in the bacterial load assays within $24-48 \mathrm{~h}$ of their collection.

For the second follow-up experiment, we set out to determine whether, under normal population culture conditions (i.e., in the absence of the experimental introduction of additional bacterial pathogens), females that differed in the number (and (or) timing) of mates also differed in their subsequent bacterial loads. It has been suggested that substantial amounts of bacteria are transmitted from males to females during mating (Miest and Bloch-Qazi 2008; but see Knell and Webberley 2004 for an opposing view), so we conducted a test to determine if the differences observed in the previous experiments may be due to differences in the number and/or timing of matings of flies. For this test, we collected $\sim 300$ virgin IV females (as above), housed them individually in vials, and randomly assigned them to one of four different male-mating treatments for which the timing and number of mating opportunities in a period of $48 \mathrm{~h}$ was manipulated. In the control group ("0-0" treatment), we kept females in isolation for the entire experimental period. In the first experimental group (treatment "1-0"), we introduced a single sexually mature IV male into a female's vial and watched them for a period of $3 \mathrm{~h}$. During that time, we scanned the vials every $5 \mathrm{~min}$ for the initiation of copulation. Once mating pairs had separated, we removed the males and kept females in isolation in the incubator for an additional $\sim 45 \mathrm{~h}$. In the second experimental group (treatment " $0-1$ "), we introduced a single sexually mature IV male into a female's vial half-way through the $48 \mathrm{~h}$ experimental period. We scanned the vials every $5 \mathrm{~min}$ for copulations for a $3 \mathrm{~h}$ period; upon the termination of copulation, we removed the males. We retained the females in the vials in isolation in the incubator for an additional $\sim 21 \mathrm{~h}$. In the third experimental group (treatment "1-1"), females were mated twice during the experimental mating period. To ensure that all females in this treatment mated on both days of the experiment, females were mated on the first day of the experiment with "spermless" males (described in Kuijper et al. 2006; Long et al. 2010). We created these males by mating virgin IV females to C(1;Y) $y^{1} B^{1} / \mathrm{O}$ males. These males lack a Y-chromosome, and while incapable of transferring sperm, do court and transfer normal amounts of seminal proteins (Ingman-Baker and Candido 1980; Chapman 1992). Females who have been mated to these spermless (XO) males will readily remate with a spermproducing (XY) male on the subsequent day (Kuijper et al. 2006; Long et al. 2010). Thus, we introduced a single IV male into the female vials at the $24 \mathrm{~h}$ point in the experimental period, which were monitored for copulations (as in other treatments), before being removed from the vials. At the end of the $48 \mathrm{~h}$ experimental period, we removed females in the four treatments from their vials, pooled them into groups of 4-5, and measured their bacterial loads (using the protocol described above).

\section{Statistical analysis}

We conducted all analyses using R version 2.15.3 (R Core Team 2012). All data and analysis code are available on the Dryad digital repository (10.5061/dryad.j2t44). To examine the effects of sire attractiveness and pathogen exposure on survivorship, we constructed Generalized Linear Models (GLM) with logit link function and quasibinomial error distribution, where the number of offspring eclosed on the 14th day from each of the experimental vials was the dependent variable and the initial number of eggs was the binomial denominator. The model included sire reproductive success ("stud" or "dud"), the presence of pathogen (yes or no), and their interaction term as independent variables. We assessed the statistical significance of each of these terms using log-likelihood ratio (LLR) $\chi^{2}$ tests 
which were implemented using the ANOVA function in the car package (Fox 2009) to compare the deviance of each model including all factors with a model excluding the effect being tested (e.g., Andrés and Arnqvist 2001).

We analyzed the bacterial loads (CFU/fly) carried by flies in the four experimental groups using a three-way ANOVA to test the significance of sire reproductive success ("stud" or "dud"), the presence of pathogen (yes or no), the sex of the flies being measured (male or female), and the interaction between these factors. Subsequently, bacterial loads were analyzed separately by sex using a two-way ANOVA to test the significance of sire reproductive success ("stud" or "dud"), the presence of pathogen (yes or no), and the interaction among these factors. Prior to these analyses, we log-transformed the bacterial load data to ensure the normality of each group's distribution (which we assessed using the Shapiro-Wilk test). Additionally, we tested the equality of variances using a Bartlett's test. Similarly, we used a two-way ANOVA to separately test the effect of sire reproductive success ("stud" or "dud"), the presence of pathogen (yes or no), and the interaction between these factors on the weight data collected from both males and females. We also calculated Cohen's $d$ effect sizes (Cohen 1988) using the function in the effsize package (Torchiano 2014) to measure the strength of the effect of sire type or pathogen presence on our response variables.

In the first of the follow-up experiments, we analyzed log-transformed bacterial loads in virgin males and females separately by sex using a two-way ANOVA to test the significance of sire reproductive success ("stud" or "dud"), the presence of pathogen (yes or no), and the interaction between these factors. We used a one-way ANOVA to compare group means of log-transformed bacterial loads between treatments in the second follow-up experiment.

\section{Results}

The experimental addition of pathogen to the vial environment resulted in a significant decrease in the egg-to-adult survivorship rate of both "stud"- and "dud"-sired offspring compared to those control vials in which $P$. aeruginosa had not been added (mean survivorship \pm SE for pathogen positive vials: $0.710 \pm 0.019$; for controls: $0.811 \pm 0.016$ ). However, sire reproductive success had no statistically significant effect on offspring survivorship (mean survivorship \pm SE for "stud" vials: $0.745 \pm 0.016$; for "dud" vials: $0.780 \pm 0.020$ ), and the interaction between sire reproductive success and pathogen exposure was also not statistically significant (Table 1).

When we weighed the adult flies, we found that males from vials that had been exposed to the pathogen were marginally lighter (Cohen's $d=0.185$ ) than those males developing in the nonpathogen vials $(P=0.065)$, whereas neither sire attractiveness nor the interaction between sire and pathogen were significant (Tables 2 and 3). For females, neither sire reproductive success, the presence/absence of pathogen, nor the interaction between these factors had a significant effect on their weight (Tables 2 and 3 ).

Table 1. Analysis of deviance conducted for a general linearized model testing the effect of sire attractiveness ("stud" or "dud") and a bacterial pathogen ("experimental" or "control") on egg-to-adult survivorship of Drosophila melanogaster.

\begin{tabular}{lrrr} 
Effect & LLR $\chi^{2}$ & df & \multicolumn{1}{c}{} \\
\hline Sire & 2.021 & 1 & 0.155 \\
Pathogen & 13.599 & 1 & $<0.001$ \\
Sire:Pathogen & 3.075 & 1 & 0.080 \\
\hline
\end{tabular}

Note: LLR, log-likelihood ratio; df, degrees of freedom. 
Table 2. Analysis of variance testing the effect of sire attractiveness ("stud" or "dud") and a bacterial pathogen ("experimental" or "control") on weights of adult male and female Drosophila melanogaster from vials developing under experimental conditions.

\begin{tabular}{|llcccc|}
\hline Sex & Factors & df & Mean square & $F$ & $P$ \\
\hline Male & & & & & \\
& Sire & 1 & 0.0004 & 1.136 & 0.288 \\
\hline & Pathogen & 1 & 0.0012 & 3.432 & 0.065 \\
\hline & Sire:Pathogen & 1 & 0.0004 & 1.221 & 0.270 \\
\hline \multirow{2}{*}{ Female } & Residuals & 235 & 0.0003 & - & - \\
\hline & & & & & 0.744 \\
\hline & Sire & 1 & 0.0004 & 0.107 & 0.320 \\
\hline & Pathogen & 1 & 0.0038 & 0.993 & 0.110 \\
\hline
\end{tabular}

Note: df, degrees of freedom.

Table 3. Summary statistics (mean \pm SE) for values of bacterial loads (logCFU/fly) and weights (mg) measured in adult male and female Drosophila melanogaster sired by males differing in attractiveness ("stud" or "dud") and developing under either "experimental" or "control" pathogen exposure conditions.

\section{Sex Sire attractiveness Pathogen exposure Bacterial load (logCFU/fly) $\quad N \quad$ Dry weight (mg)}

\section{Male}

$\begin{array}{llrrr}\text { "stud" } & \text { Experimental } & 3.691 \pm 0.171 & 9 & 0.1684 \pm 0.0035 \\ \text { "stud" } & \text { Control } & 4.262 \pm 0.217 & 8 & 0.1754 \pm 0.0038 \\ \text { "dud" } & \text { Experimental } & 4.510 \pm 0.142 & 10 & 0.1685 \pm 0.0029 \\ \text { "dud" } & \text { Control } & 4.429 \pm 0.120 & 10 & 0.1703 \pm 0.0033\end{array}$

\begin{tabular}{rllll} 
Female & & & & \\
"stud" & Experimental & $4.467 \pm 0.198$ & 9 & $0.3290 \pm 0.0115$ \\
"stud" & Control & $4.885 \pm 0.109$ & 9 & $0.3081 \pm 0.0111$ \\
"dud" & Experimental & $4.154 \pm 0.263$ & 9 & $0.3136 \pm 0.0112$ \\
"dud" & Control & $4.343 \pm 0.192$ & 7 & $0.3183 \pm 0.0112$ \\
\hline
\end{tabular}

Note: $N$ indicates the number of vials from each combination of treatments (out of 10) that yielded usable bacterial load values. CFU, colony forming units.

In our initial three-way analysis of the effect of sex, sire, and pathogen exposure, we observed that a fly's bacterial load was strongly influenced by both their sex and the type of male that had sired them (Tables 3 and 4 ). The nature of this interaction became evident when the sexes were analyzed separately: sire attractiveness had a significant effect on the size of the bacterial load carried in both sons $(P=0.003)$ and daughters $(P=0.036$; Tables 3 and 4$)$, but not in the same manner. Sons sired by "stud" fathers carried a smaller bacterial load compared to sons sired by "dud" fathers (Fig. 1; Cohen's $d=0.982$ ). In contrast, the daughters sired by "stud" fathers carried a larger bacterial load then those daughters sired by "dud"-fathers (Fig. 1; Cohen's $d=0.442$ ). 
Table 4. Analysis of variance testing the effect of sire attractiveness ("stud" or "dud") and a bacterial pathogen "experimental" or "control") on bacterial loads (logCFU/fly) of adult male and female Drosophila melanogaster from vials developing under experimental conditions.

\begin{tabular}{|c|c|c|c|c|c|}
\hline Sex & Factors & df & Mean square & $F$ & $P$ \\
\hline \multicolumn{6}{|c|}{ Both } \\
\hline & Sex & 1 & 0.972 & 3.389 & 0.070 \\
\hline & Sire & 1 & 0.053 & 0.183 & 0.670 \\
\hline & Pathogen & 1 & 1.425 & 4.967 & 0.029 \\
\hline & Sex:Sire & 1 & 3.762 & 13.118 & $<0.001$ \\
\hline & Sex:Pathogen & 1 & 0.038 & 0.134 & 0.716 \\
\hline & Sire:Pathogen & 1 & 0.890 & 3.102 & 0.083 \\
\hline & Sex:Sire:Pathogen & 1 & 0.196 & 0.682 & 0.412 \\
\hline & Residuals & 63 & 0.287 & - & - \\
\hline \multicolumn{6}{|c|}{ Male } \\
\hline & Sire & 1 & 2.387 & 10.01 & 0.003 \\
\hline & Pathogen & 1 & 0.439 & 1.839 & 0.184 \\
\hline & Sire:Pathogen & 1 & 0.975 & 4.086 & 0.051 \\
\hline & Residuals & 33 & 0.239 & - & - \\
\hline \multicolumn{6}{|c|}{ Female } \\
\hline & Sire & 1 & 1.634 & 4.810 & 0.036 \\
\hline & Pathogen & 1 & 0.817 & 2.405 & 0.131 \\
\hline & Sire:Pathogen & 1 & 0.111 & 0.325 & 0.573 \\
\hline & Residuals & 30 & 0.340 & - & - \\
\hline
\end{tabular}

Note: CFU, colony forming units; df, degrees of freedom.

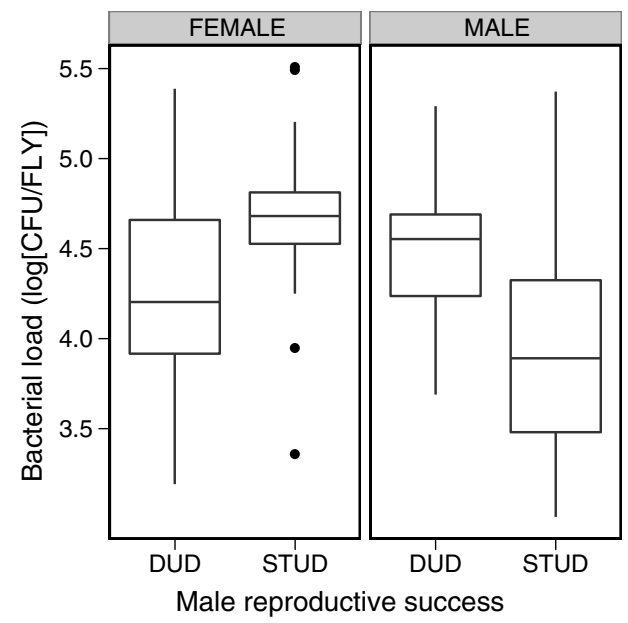

Fig. 1. Boxplots of bacterial loads of female and male Drosophila melanogaster adults that had been sired by males of differing attractiveness. The box encloses values between the first and third quartiles of the data (the inter-quartile range (IQR)), whereas the horizontal bar within the box indicates the median. Whiskers extend from the box to largest/smallest values that are within $1.5 \times$ the IQR of the box. Values outside that range are outliers and are indicated by circles. 
In the first follow-up experiment (of male and female adult virgin offspring), neither of the main effects, nor their interaction, were statistically significant for either the females (sire; $F_{1,41}=0.01$, $P=0.91$; pathogen exposure: $F_{1,41}=0.002, P=0.96$; sire $\times$ pathogen exposure: $F_{1,41}=3.44$, $P=0.07$; Supplementary Material 2) or for males (sire; $F_{1,25}=1.77, P=0.20$; pathogen exposure: $F_{1,25}=0.42, P=0.52$; sire $\times$ pathogen exposure: $F_{1,25}=1.68, P=0.21$; Supplementary Material 3). In the second follow-up experiment (involving assays of females that differed only in the timing and (or) number of matings), there was no effect of treatment on the mean bacterial loads $\left(F_{3,45}=0.07\right.$, $P=0.98$, Supplementary Material 4).

\section{Discussion}

Ever since Darwin (1871) proposed the theory of sexual selection to explain the evolution of elaborate display traits in males, biologists have attempted to understand the extent to which the selective pressures produced from differential mating success complement, or conflict, with the pressures resulting from natural selection (Andersson 1994; Arnqvist and Rowe 2005). In this study, by experimentally mating females with males that had been previously assayed as being of relatively high or low sexual attractiveness and (or) competitiveness (i.e., "studs" or "duds"), we were able to measure the cross-generational effects associated with sexual selection. We found that differences in the attractiveness/competitiveness of potential males was related to observed differences in the bacterial loads carried by their offspring, but this effect ultimately depended on the sex of the individual fly in which these genes were expressed.

Overall, the addition of the bacteria to vials containing D. melanogaster eggs resulted in an egg-to-adult survivorship rate that was lower than that observed in the control vials. Furthermore, males eclosing from the experimental vials were marginally smaller than those that had developed in the control vials. These results highlight the fact that $P$. aeruginosa is a known pathogen of Drosophila (Boman et al. 1972; D'Argenio et al. 2001), and is consistent with a previous study (Young et al. 2009) that used the same strain of bacteria and means of infection that reported a similar negative effect on survivorship. Kraaijeveld et al. (2001) hypothesized that the common embryonic origin of the head muscles (used in feeding) and the hemopoietic organ (that produces the hemocytes that are responsible for the cellular immune response) may provide a proximate explanation for any developmental trade-offs in Drosophila. Thus, the relatively smaller size of males in the presence of pathogens is consistent with previous work that has found that flies experimentally evolved in an immunologically challenging environment also exhibited lower feeding rates (Fellowes et al. 1999), and that there are developmental trade-offs between growth and immunocompetence (Modak et al. 2009). The fact that this phenomenon was only observed in male offspring may reflect the effects of sex-specific selective pressures. It has been repeatedly suggested (Trivers 1972; Zuk 1990; Rolff 2002) that a male's lifetime reproductive success may depend more on the attraction of mates than it does for females, where variation in fitness is often strongly correlated with body size, as larger females have more resources to invest in fecundity (Lefranc and Bundgaard 2000; Pitnick and García-González 2002; Long et al. 2009). Thus, the sexes may be under divergent selection over the allocation of limited resources; this is a promising avenue for future research.

Sex-specific responses to pathogen exposure were also observed in our assay of bacterial loads. While the sons of "stud" males carried fewer bacteria than did those sired by "dud" fathers, the opposite pattern was observed in daughters, where those sired by "duds" had smaller bacterial loads. This pattern of sire attractiveness-linked immunocompetence observed in sons is partially consistent with the theoretical predictions initially made by Hamilton and Zuk (1982), as well as the empirical observations of Rolff and Kraaijeveld (2003): that females are selecting to mate with males for their superior immune response alleles. These effects may be further exaggerated if the magnitude of maternal effects 
(or paternity-induced effects) differed between the mothers mated to "studs" or "duds" (e.g., Kotiaho et al. 2003). However, the gains experienced by the sons sired by "stud" males are not shared with their sisters, who carried relatively high bacterial loads. Such a pattern is suggestive of the presence of sexually antagonistic allelic variation, whereby the effect of an allele on Darwinian fitness ultimately depends on the sex in which it is expressed (Chippindale et al. 2001; Arnqvist and Rowe 2005; Pischedda and Chippindale 2006). This type of genetic conflict arises because, while most of the genome is shared between the sexes, the phenotypes and life-histories associated with fitness-maximization are often quite different between males and females (Holland and Rice 1998). As the development, maintenance, and utilization of an immune system are costly (Rolff and Siva-Jothy 2003; Kraajijeveld and Wertheim 2009), and an individual's available resources are finite, it is likely that conflicts over investment should arise between the sexes (Rolff 2002). Specifically, it has been hypothesized that females will be selected to invest more heavily into immune function, as a prolonged lifespan will increase the opportunity to produce offspring, whereas in males, variance in fitness is not associated with longevity, but instead with mating rate, and consequently may be selected to bias resource investment away from immune function and toward other fitness-maximizing traits (Rolff 2002; Rolff et al. 2005). This hypothesis is supported by evidence of systematic female biases in the expression of some immune function traits in insects, indicative of a history of divergent selection on the sexes (Nunn et al. 2009). It is our conjecture that our observations are consistent with the effects of sexually antagonistic genetic variation acting on both individual physiological "condition" (sensu Rowe and Houle 1996) and the allocation of resources toward immune defence. When "studs" sire sons, they contribute alleles that may produce phenotypes of superior (male-benefit) physiological condition, and relatively lower allocation toward immune investment. However, because these individuals are in a relatively good physiological state, they have more resources to allocate to growth, development, and maintenance needs, allowing them to withstand bacterial challenges. Meanwhile, the sons of "duds" end up in worse physiological state, with fewer resources to allocate to all traits (including immunity). As such, they are less able to mount a defence against pathogens. The patterns of bacterial loads observed in females are also consistent with the effects of sexually antagonistic alleles. Females sired by "studs" inherit many "male-benefit" alleles from their fathers, which are deleterious when expressed in a female genetic background. Daughters of "studs" may be expressing alleles that divert resources away from immune function, as well as other alleles (e.g., for locomotory behaviour, as in Long and Rice 2007) that result in a relatively inferior physiological condition. Consequently, these females may be less capable of mounting an immune response, manifested as higher bacterial loads. In the case of daughters of "duds", these females have inherited alleles that - while at a selective disadvantage when expressed in males, are beneficial when expressed in females. These females may be in a better physiological state and able to allocate more resources to their immune system, resulting in relatively lower bacterial counts. Ultimately, such sexually antagonistic alleles contribute to the so-called "gender load" (Rice and Chippindale 2002) in sexually reproducing species, as their presence interferes with the action of selection to remove deleterious alleles from the gene pool, and ultimately depresses the fitness of the population.

Our results are consistent with the recent findings of Vincent and Sharp (2014), who assayed the immunity of 50 homozygous lines of $D$. melanogaster exposed to $P$. aeruginosa and also found evidence of sexual antagonism in the patterns of resistance and tolerance to the bacterial pathogen expressed by male and female genotypes. More broadly, our study joins a growing body of research on the link between immune and reproductive traits, and how selection may shape their expression in both males and females. While the specific environment, selective pressures, and underlying genetic architecture of traits in our lab-reared IV population of D. melanogaster are unlikely to be directly comparable to other study systems, our results highlight the potential for sex-specific selection to shape evolution of immunocompetence in many other species. For instance, in the beetle, Callosobruchus maculatus, both males and females raised under male-biased sex ratios exhibited a 
decrease in the investment in antibacterial immune function (measured as lytic activity) compared to individuals from populations evolving under female-biased sex ratios (van Lieshout et al. 2014). Increased sexual selection acting on males resulting from heightened competition/conflict may have led to the fixation of male-benefit alleles (and by corollary the loss of female-benefit alleles) from the gene pool, and subsequently resulted in an evolutionary a shift toward phenotypes in which fewer resources are allocated toward immune function. It is possible that this may have also occurred in McKean and Nunney's (2008) study of D. melanogaster, where they observed decreases immunological resistance in flies from populations experimentally evolving under male-biased sex ratios. In the yellow dung fly, Scathophaga stercoraria, the experimental removal of sexual conflict (via enforced monogamy) for multiple generations led to the evolution of females capable of exhibiting a greater immune response compared to those from polyandrous lines (Hosken 2001). Thus, depending on the magnitude of the selective pressures acting on males and females, different levels (and dimorphisms) of investment into immunocompetence can evolve (see Vincent and Gwynne 2014). Furthermore, our study complements previous studies (e.g., Fedorka and Mousseau 2004; Oneal et al. 2007) that have reported distinct sex-specific benefits/costs associated with female mating biases, by highlighting an important fitness-related trait (immunocompetence) that may be associated with differences in offspring competitive success. Integrating information on sex-specific fitness-maximizing strategies, pathogenic risks, developmental costs, and resource availabilities in both males and females should lead to better understanding inter- and intra-specific diversity in immune and reproductive traits.

It is worth noting at this juncture that our initial estimates of bacterial load were based on counts made from adult flies that were $\sim 14$ days old ( $\sim 4-5$ days post-eclosion), collected from their natal vial. As such, the adults that we assayed had been living in vials containing waste products, a rich microbial community and were likely to have been (multiply-) mated. To better understand the differences in the bacterial loads of "stud"- and "dud"-sired male and female offspring, and the role of mating, we subsequently conducted two complementary follow-up experiments designed to shed light on potential factors that might have given rise to our observed patterns. In the first of these assays, we set out to determine if the differences in bacterial loads seen in mated, adult flies assayed 4-5 days after their eclosion were also apparent earlier in their life cycle. As such, we measured bacterial loads in newly eclosed (virgin) flies, and found no differences between those sired by "studs" and those sired by "duds". This suggests that the effects of immunity-related genes associated with differential bacterial load that are linked to sire mating success may not be manifested phenotypically until later in their adulthood. This hypothesis is consistent with previous work by Chippindale et al. (2001) and Gibson et al. (2002) in which it is suggested that sexually antagonistic alleles in Drosophila are not expressed during the juvenile part of the life cycle, in which the sex roles are most similar (but see Perry et al. 2014). It remains to be seen how (or if) the differences in immunocompetence phenotypes associated with paternal reproductive success in the presence or absence of a pathogen translate into differences in individual reproductive success in males and (or) females. Empirical measurements of offspring lifetime reproductive success will help shed light on the role that sexual selection plays in the evolution of immunocompetence, and the likelihood of resolution of any underlying conflict over this trait.

In addition to experiencing immunological challenges from the bacteria-laden media of their natal vial environment, it has been suggested that males transmit bacteria to their mates during mating (Miest and Bloch-Qazi 2008). Furthermore, the physical damage incurred by females during copulation (Kamimura 2007) might provide additional avenues for infection. If so, the differences seen between "stud"- and "dud"-sired daughters may reflect differences in the number and (or) types of males mated. If "stud" males do possess alleles favouring the high mating rate phenotype, and there is both sexual conflict and an intersexual correlation between the sexes for this trait (as 
suggested by Holland and Rice 1998), then it may be that daughters of "studs" end up with greater bacterial loads due to a relatively higher mating rate or differences in the types of males with which they meet. To begin to test this hypothesis, we experimentally mated females to differing numbers of males and (or) at different times and compared their bacterial loads to virgin females of the same age. Our test failed to detect any significant differences between these groups. While Miest and Bloch-Qazi (2008) did find that males could transfer bacteria to their mates during mating, their protocol involved dipping a male's abdomen into a bacterial broth before mating, which may have increased the likelihood of detecting a successful transfer. Other studies (reviewed in Chapman 2001; Lawniczak et al. 2007) have found that that several of the compounds that make up the seminal fluid of D. melanogaster males have protective antimicrobial properties (also see Mueller et al. 2007). Furthermore, Peng et al. (2005) report that the innate immune system of females is stimulated by the presence of the seminal peptide, Sex-peptide, which might have mediated any potential change in bacterial loads in the mated flies. It should be noted that this assay was conducted in vials containing fresh media, which was more sterile than typical culture conditions, and that we did not measure the mating success of the fathers of our target females. Future studies of bacterial loads on flies in which age, developmental environment, and (or) the number of mating partners as well as parental mating success may provide a better understanding of the nature of this sex-linked variation in immunocompetence.

In summary, we provide evidence that there are important immunological consequences associated with mate-choice outcomes in the model species, Drosophila melanogaster. In our study system, offspring produced by females who mate with reproductively successful males display greater immunocompetence, but this pattern is confined to sons, and is offset by immunological disadvantages experienced in their daughters. This pattern may reflect the operation of sexually antagonistic alleles over optimal allocation to the immune system. Our post hoc experiments suggest that the differences in bacterial loads observed between "stud" and "dud"-sired offspring are not evident upon their eclosion as adults, but become more apparent over time, and that the differences in daughters may not be due to differences in their mating rates. Ultimately, the extent to which these differences contribute to variation in female lifetime reproductive success will determine the strength and direction of sexual selection, and shape our understanding of the evolution of life history traits in both sexes.

\section{Acknowledgements}

We thank H. Tennant, L. DeJong, E.M. Martin, C. Russell, J. Tzaponis, K. Schang, K. Seaver, T. Balasubramaniam, E. Razvi, R. Tutulan, D. MacNeil, E. Wilson, and T. Brenner for their support and assistance with assays, as well as R. Slawson, M. Costea and J. McGeer for the generous use of their equipment. N.B. Gallo, J. Rutherford, K. Stevens, and several anonymous reviewers are thanked for their feedback on this manuscript. This work was supported by Natural Sciences and Engineering Council of Canada (NSERC) Discovery Grants held by TAFL and JW as well as additional funding from WLU's Faculty of Science Students Association.

\section{Author contributions}

Conceived and designed the study: AG, TB, KP, JW, TAFL. Performed the experiments/collected the data: AG, TB, KP, JW, TAFL. Analyzed and interpreted the data: AG, TB, KP, JW, TAFL. Contributed resources: JW, TAFL. Drafted or revised the manuscript: AG, TB, KP, JW, TAFL.

\section{Competing interests}

The authors have declared that no competing interests exist. 


\section{Data accessibility statement}

All relevant data are within the paper, in the Supplementary Material, and in the Dryad Digital Repository (doi:10.5061/dryad.j2t44).

\section{Supplementary material}

The following Supplementary Material is available with the article through the journal website at doi:10.1139/facets-2015-0007.

Supplementary Material 1

Supplementary Material 2

Supplementary Material 3

Supplementary Material 4

\section{References}

Andersson M. 1994. Sexual selection. 1st edition. Princeton University Press, Princeton, New Jersey.

Andrés JA, and Arnqvist G. 2001. Genetic divergence of the seminal signal-receptor system in houseflies: the footprints of sexually antagonistic coevolution? Proceedings of the Royal Society of London Series B, 268: 399-405. doi:10.1098/rspb.2000.1392.

Apidianakis Y, and Rahme LG. 2009. Drosophila melanogaster as a model host for studying Pseudomonas aeruginosa infection. Nature Protocols, 4: 1285-1294. PMID:19680242. doi:10.1038/ nprot.2009.124.

Arnqvist G, and Rowe L. 2005. Sexual conflict. Princeton University Press, Princeton, New Jersey.

Ashburner M. 1989. Drosophila: a laboratory handbook. Cold Spring Harbor Laboratory Press, Cold Spring Harbor, New York.

Boman HG, Nilsson I, and Rasmuson B. 1972. Inducible antibacterial defence system in Drosophila. Nature, 237: 232-235. PMID:4625204. doi:10.1038/237232a0.

Broderick NA, and Lemaitre B. 2009. Recognition and response to microbial infection in Drosophila. In Insect infection and immunity: evolution, ecology, and mechanisms. Edited by J Rolff and S Reynolds. Oxford University Press, Oxford, UK. pp. 13-33.

Chandler JA, Lang JM, Bhatnagar S, Eisen JA, and Kopp A. 2011. Bacterial communities of diverse Drosophila species: ecological context of a host-microbe model system. PLoS Genetics, 7: e1002272. PMID:21966276. doi:10.1371/journal.pgen.1002272.

Chapman T. 1992. A cost of mating with males that do not transfer sperm in female Drosophila melanogaster. Journal of Insect Physiology, 38: 223-227. doi:10.1016/0022-1910(92)90070-T.

Chapman T. 2001. Seminal fluid-mediated fitness traits in Drosophila. Heredity, 87: 511-521. PMID:11869341. doi:10.1046/j.1365-2540.2001.00961.x.

Chippindale AK, Alipaz JA, Chen HW, and Rose MR. 1997. Experimental evolution of accelerated development in Drosophila. 1. Developmental speed and larval survival. Evolution, 51: 1536-1551. doi: $10.2307 / 2411206$. 
Chippindale AK, Gibson JR, and Rice WR. 2001. Negative genetic correlation for adult fitness between sexes reveals ontogenetic conflict in Drosophila. Proceedings of the National Academy of Sciences USA, 98: 1671-1675. doi:10.1073/pnas.98.4.1671.

Cohen J. 1988. Statistical power analysis for the behavioral sciences. 2nd edition. Academic Press, New York, New York.

D’Argenio DA, Gallagher LA, Berg CA, and Manoil C. 2001. Drosophila as a model host for Pseudomonas aeruginosa infection. Journal of Bacteriology, 183: 1466-1471. doi:10.1128/ JB.183.4.1466-1471.2001.

Darwin C. 1871. The descent of man, and selection in relation to sex. John Murray, London, UK.

Fedorka KM, and Mousseau TA. 2004. Female mating bias results in conflicting sex-specific offspring fitness. Nature, 429: 65-67. PMID:15129280. doi:10.1038/nature02492.

Fellowes MDE, Kraaijeveld AR, and Godfray HCJ. 1999. Association between feeding rate and parasitoid resistance in Drosophila melanogaster. Evolution, 53: 1302-1305. doi:10.2307/ 2640834.

Folstad I, and Karter AJ. 1992. Parasites, bright males, and the immunocompetence handicap. American Naturalist, 139: 603-622. doi:10.1086/285346.

Fox J. 2009. car: companion to applied regression. R package version 1.2-16 [online]: Available from http://CRAN.R-project.org/package=car.

Gibson JR, Chippindale AK, and Rice WR. 2002. The X chromosome is a hot spot for sexually antagonistic fitness variation. Proceedings of the Royal Society of London Series B, 269: 499-505. PMID:11886642. doi:10.1098/rspb.2001.1863.

Greenspan RJ, and Ferveur J-F. 2000. Courtship in Drosophila. Annual Review of Genetics, 34: 205-232. PMID:11092827. doi:10.1146/annurev.genet.34.1.205.

Hall JC. 1994. The mating of a fly. Science, 264: 1702-1714. PMID:8209251. doi:10.1126/ science.8209251.

Hamilton WD, and Zuk M. 1982. Heritable true fitness and bright birds: a role for parasites? Science, 218: 384-387. PMID:7123238. doi:10.1126/science.7123238.

Hihara F. 1981. Effects of the male accessory gland secretion on oviposition and remating in females of Drosophila melanogaster. Zoological Magazine, 90: 307-316.

Hoffmann JA. 2003. The immune response of Drosophila. Nature, 426: 33-38. PMID:14603309. doi:10.1038/nature02021.

Holland B, and Rice WR. 1998. Perspective: chase-away sexual selection: antagonistic seduction versus resistance. Evolution, 52: 1-7. doi:10.2307/2410914.

Hosken DJ. 2001. Sex and death: microevolutionary trade-offs between reproductive and immune investment in dung flies. Current Biology, 11: R379-R380. PMID:11378399. doi:10.1016/ S0960-9822(01)00211-1.

Houle D. 1991. Genetic covariance of fitness correlates: what genetic correlations are made of and why it matters. Evolution, 45: 630-648. doi:10.2307/2409916. 
Ingman-Baker J, and Candido EPM. 1980. Proteins of the Drosophila melanogaster male reproductive system: two-dimensional gel patterns of proteins synthesized in the XO, XY, and XYY testis and paragonial gland and evidence that the $\mathrm{Y}$ chromosome does not code for structural sperm proteins. Biochemical Genetics, 18: 809-828. PMID:6781473. doi:10.1007/BF00484595.

Kamimura Y. 2007. Twin intromittent organs of Drosophila for traumatic insemination. Biology Letters, 3: 401-404. PMID:17519186. doi:10.1098/rsbl.2007.0192.

Khush RS, and Lemaitre B. 2000. Genes that fight infection: what the Drosophila genome says about animal immunity. Trends in Genetics, 16: 442-449. PMID:11050330. doi:10.1016/S0168-9525(00)02095-3.

Kirkpatrick M, and Ryan MJ. 1991. The evolution of mating preferences and the paradox of the lek. Nature, 350: 33-38. doi:10.1038/350033a0.

Knell RJ, and Webberley KM. 2004. Sexually transmitted diseases of insects: distribution, evolution, ecology and host behavior. Biological Reviews, 79: 557-581. PMID:15366763. doi:10.1017/ S1464793103006365.

Kokko H, Jennions MD, and Brooks R. 2006. Unifying and testing models of sexual selection. Annual Review of Ecology, Evolution, and Systematics, 37: 43-66. doi:10.1146/annurev. ecolsys.37.091305.110259.

Kotiaho JS, Simmons LW, Hunt J, and Tomkins JL. 2003. Males influence maternal effects that promote sexual selection: a quantitative genetic experiment with dung beetles Onthophagus taurus. American Naturalist, 161: 852-859. doi:10.1086/375173.

Kraaijeveld AR, and Godfray HCJ. 1997. Trade-off between parasitoid resistance and larval competitive ability in Drosophila melanogaster. Nature, 389: 278-280. PMID:9305840. doi:10.1038/38483.

Kraaijeveld AR, Limentani EC, and Godfray HCJ. 2001. Basis of the trade-off between parasitoid resistance and larval competitive ability in Drosophila melanogaster. Proceedings of the Royal Society of London Series B, 268: 259-261. PMID:11217895. doi:10.1098/rspb.2000.1354.

Kraaijeveld AR, and Wertheim B. 2009. Costs and genomic aspects of Drosophila immunity to parasites and pathogens. In Insect infection and immunity: evolution, ecology, and mechanisms. Edited by J Rolff and S Reynolds. Oxford University Press, Oxford, UK. pp. 187-205.

Kuijper B, Stewart AD, and Rice WR. 2006. The cost of mating rises nonlinearly with copulation frequency in a laboratory population of Drosophila melanogaster. Journal of Evolutionary Biology, 19: 1795-1802. PMID:17040376. doi:10.1111/j.1420-9101.2006.01186.x.

Kurtz J, and Sauer KP. 1999. The immunocompetence handicap hypothesis: testing the genetic predictions. Proceedings of the Royal Society of London Series B, 266: 2515-2522. PMID:10693823. doi:10.1098/rspb.1999.0954.

Lawniczak MK, Barnes AI, Linklater JR, Boone JM, Wigby S, and Chapman T. 2007. Mating and immunity in invertebrates. Trends in Ecology \& Evolution, 22: 48-55. PMID:17028056. doi:10.1016/j.tree.2006.09.012.

Lefranc A, and Bundgaard J. 2000. The influence of male and female body size on copulation duration and fecundity in Drosophila melanogaster. Hereditas, 132: 243-247. PMID:11075519. doi:10.1111/ j.1601-5223.2000.00243.x. 
Long TAF, and Rice WR. 2007. Adult locomotory activity mediates intralocus sexual conflict in a laboratory-adapted population of Drosophila melanogaster. Proceedings of the Royal Society of London B: Biological Sciences, 274: 3105-3112. doi:10.1098/rspb.2007.1140.

Long TAF, Montgomerie R, and Chippindale AK. 2006. Quantifying the gender load: can population crosses reveal interlocus sexual conflict? Philosophical Transactions of the Royal Society of London Series B, 361: 363-374. PMID:16612894. doi:10.1098/rstb.2005.1786.

Long TAF, Pischedda A, Nichols RV, and Rice WR. 2010. The timing of mating influences reproductive success in Drosophila melanogaster: implications for sexual conflict. Journal of Evolutionary Biology, 23: 1024-1032. PMID:20345814. doi:10.1111/j.1420-9101.2010.01973.x.

Long TAF, Pischedda A, Stewart AD, and Rice WR. 2009. A cost of sexual attractiveness to highfitness females. PLoS Biology, 7: e1000254. PMID:19997646. doi:10.1371/journal.pbio.1000254.

Markow TA, Quaid M, and Kerr S. 1978. Male mating experience and competitive courtship success in Drosophila melanogaster. Nature, 276: 821-822. doi:10.1038/276821a0.

McKean KA, and Nunney L. 2008. Sexual selection and immune function in Drosophila melanogaster. Evolution, 62: 386-400. PMID:18070086. doi:10.1111/j.1558-5646.2007.00286.x.

Miest TS, and Bloch-Qazi MC. 2008. Sick of mating: sexual transmission of a pathogenic bacterium in Drosophila melanogaster. Fly, 2: 215-219. PMID:18769140. doi:10.4161/fly.6726.

Modak SG, Satish KM, Mohan J, Dey S, Raghavendra N, Shakarad M, et al. 2009. A possible tradeoff between developmental rate and pathogen resistance in Drosophila melanogaster. Journal of Genetics, 88: 253-256. PMID:19700866. doi:10.1007/s12041-009-0036-8.

Mueller JL, Page JL, and Wolfner MF. 2007. An ectopic expression screen reveals the protective and toxic effects of Drosophila seminal fluid proteins. Genetics, 175: 777-783. PMID:17110486. doi:10.1534/genetics.106.065318.

Nunn CL, Lindenfors P, Pursall ER, and Rolff J. 2009. On sexual dimorphism in immune function. Philosophical Transactions of the Royal Society of London Series B, 364: 61-69. PMID:18926977. doi:10.1098/rstb.2008.0148.

Oneal E, Connallon T, and Knowles LL. 2007. Conflict between direct and indirect benefits of female choice in desert Drosophila. Biology Letters, 3: 29-32. doi:10.1098/rsbl.2006.0565.

Partridge L, Hoffmann A, and Jones JS. 1987. Male size and mating success in Drosophila melanogaster and D. pseudoobscura under field conditions. Animal Behaviour, 35: 468-476. doi:10.1016/ S0003-3472(87)80272-5.

Peng J, Zipperlen P, and Kubli E. 2005. Drosophila sex-peptide stimulates female innate immune system after mating via the Toll and Imd pathways. Current Biology, 15: 1690-1694. PMID:16169493. doi:10.1016/j.cub.2005.08.048.

Perry JC, Harrison PW, and Mank JE. 2014. The ontogeny and evolution of sex-biased gene expression in Drosophila melanogaster. Molecular Biology and Evolution, 31: 1206-1219. PMID:24526011. doi:10.1093/molbev/msu072.

Pischedda A, and Chippindale AK. 2006. Intralocus sexual conflict diminishes the benefits of sexual selection. PLoS Biology, 4: e356. PMID:17105343. doi:10.1371/journal.pbio.0040356. 
Pitnick S, and García-González F. 2002. Harm to females increases with male body size in Drosophila melanogaster. Proceedings of the Royal Society of London Series B, 269: 1821-1828. PMID:12350270. doi:10.1098/rspb.2002.2090.

Qvarnström A, Brommer JE, and Gustafsson L. 2006. Testing the genetics underlying the co-evolution of mate choice and ornament in the wild. Nature, 441: 84-86. doi:10.1038/ nature 04564 .

R Core Team. 2012. R: a language and environment for statistical computing. R Foundation for Statistical Computing, Vienna, Austria [online]: Available from https://www.R-project.org/.

Rice WR, and Chippindale AK. 2002. The evolution of hybrid infertility: perpetual coevolution between gender-specific and sexually antagonistic genes. In Genetics of mate choice: from sexual selection to sexual isolation. Edited by WJ Etges and MA Noor. Springer, New York, New York. pp. $179-188$.

Rolff J. 2002. Bateman's principle and immunity. Proceedings of the Royal Society of London Series B, 269: 867-872. PMID:11958720. doi:10.1098/rspb.2002.1959.

Rolff J, and Kraaijeveld AR. 2003. Selection for parasitoid resistance alters mating success in Drosophila. Proceedings of the Royal Society of London Series B, 270(Supplement 2): S154-S155. doi:10.1098/rsbl.2003.0024.

Rolff J, and Siva-Jothy MT. 2003. Invertebrate ecological immunology. Science, 301: 472-475. PMID:12881560. doi:10.1126/science.1080623.

Rolff J, Armitage SAO, and Coltman DW. 2005. Genetic constraints and sexual dimorphism in immune defense. Evolution, 59: 1844-1850. PMID:16329251. doi:10.1111/j.0014-3820.2005.tb01831.x.

Rose MR. 1984. Laboratory evolution of postponed senescence in Drosophila melanogaster. Evolution, 38: 1004-1010. doi:10.2307/2408434.

Rowe L, and Houle D. 1996. The lek paradox and the capture of genetic variance by condition dependent traits. Proceedings of the Royal Society of London Series B, 263: 1415-1421. doi:10.1098/ rspb.1996.0207.

Rundle HD, Ödeen A, and Mooers AØ. 2007. An experimental test for indirect benefits in Drosophila melanogaster. BMC Evolutionary Biology, 7: 36. PMID:17349042. doi:10.1186/1471-2148-7-36.

Ryan KJ, and Ray CG. 1994. Medical microbiology. Appleton \& Lange, Norwalk, Connecticut.

Sheldon BC, and Verhulst S. 1996. Ecological immunology: costly parasite defences and trade-offs in evolutionary ecology. Trends in Ecology \& Evolution, 11: 317-321. PMID:21237861. doi:10.1016/ 0169-5347(96)10039-2.

Sirot LK, Buehner NA, Fiumera AC, and Wolfner MF. 2009. Seminal fluid protein depletion and replenishment in the fruit fly, Drosophila melanogaster: an ELISA-based method for tracking individual ejaculates. Behavioral Ecology and Sociobiology, 63: 1505-1513. doi:10.1007/ s00265-009-0806-6.

Staubach F, Baines JF, Künzel S, Bik EM, and Petrov DA. 2013. Host species and environmental effects on bacterial communities associated with Drosophila in the laboratory and in the natural environment. PLoS ONE, 8: e70749. PMID:23967097. doi:10.1371/journal.pone.0070749. 
Sullivan W, Ashburner M, and Hawley RS. 2000. Drosophila protocols. Cold Spring Harbor Laboratory Press, Cold Spring Harbor, New York.

Torchiano M. 2014. effsize: Efficient effect size computation. R package version 0.5.2 [online]: Available from http://CRAN.R-project.org/package=effsize

Trivers RL. 1972. Parental investment and sexual selection. In Sexual selection and the descent of man 1871-1971. Edited by B Campbell. Aldine Publishing Co., Chicago, Illinois. pp. 136-179.

van Lieshout E, McNamara KB, and Simmons LW. 2014. Rapid loss of behavioral plasticity and immunocompetence under intense sexual selection. Evolution, 68: 2550-2558. PMID:24724572. doi:10.1111/evo.12422.

van Noordwijk AJ, and de Jong G. 1986. Acquisition and allocation of resources: their influence on variation in life history tactics. The American Naturalist, 128: 137-142. doi:10.1086/284547.

Vincent CM, and Gwynne DT. 2014. Sex-biased immunity is driven by relative differences in reproductive investment. Proceedings of the Royal Society of London B: Biological Sciences, 281: 20140333. doi:10.1098/rspb.2014.0333.

Vincent CM, and Sharp NP. 2014. Sexual antagonism for resistance and tolerance to infection in Drosophila melanogaster. Proceedings of the Royal Society of London Series B, 281: 20140987. PMID:24966317. doi:10.1098/rspb.2014.0987.

Westneat DF, and Birkhead TR. 1998. Alternative hypotheses linking the immune system and mate choice for good genes. Proceedings of the Royal Society of London Series B, 265: 1065-1073. doi:10.1098/rspb.1998.0400.

Young JA, Yourth CP, and Agrawal AF. 2009. The effect of pathogens on selection against deleterious mutations in Drosophila melanogaster. Journal of Evolutionary Biology, 22: 2125-2129. doi:10.1111/ j.1420-9101.2009.01830.x.

Zahavi A. 1975. Mate selection-a selection for a handicap. Journal of Theoretical Biology, 53: 205-214. PMID:1195756. doi:10.1016/0022-5193(75)90111-3.

Zera AJ, and Harshman LG. 2009. Laboratory selection studies of life history physiology in insects. In Experimental evolution: concepts, methods, and applications. Edited by T Garland and MR Rose. University of California Press, Berkley, California. pp. 217-262.

Zuk M. 1990. Reproductive strategies and disease susceptibility: an evolutionary viewpoint. Parasitology Today, 6(7): 231-233. PMID:15463350. doi:10.1016/0169-4758(90)90202-F. 